\title{
Measurement perspective, process, and the pandemic
}

\author{
Vadim Keyser $^{1}$ (1) $\cdot$ Hannah Howland ${ }^{2}$
}

Received: 30 October 2019 / Accepted: 18 September 2020 /Published online: 21 November 2020

(C) Springer Nature B.V. 2020

\begin{abstract}
This discussion centers on two desiderata: the role of measurement in informationgathering and physical interaction in scientific practice. By taking inspiration from van Fraassen's (2008) view, we present a methodological account of perspectival measurement that addresses empirical practice where there is complex intervention, disagreeing results, and limited theory. The specific aim of our account is to provide a methodological prescription for developing measurement processes in the context of limited theory. The account should be useful to philosophers of science, who are interested in the intersection between representation and intervention; scientists, who are interested in methodological suggestions for theory-development and reliability; and interdisciplinary researchers, who are interested in the intersections between the pandemic, built environments, and social processes. We apply the process-view of measurement to COVID-19, specifically, measuring replication in the SARS-CoV-2 virus. The aim is to show that our account tracks key elements-manipulation/intervention, independence, invariance, and theory-development-by organizing unfolding measurement processes. Additionally, we use our account to make prescriptive suggestions for measurement practice in the COVID-19 context by discussing the need to broaden measurement perspective on interaction, manipulation, and production.
\end{abstract}

Keywords Measurement theory $\cdot$ Process-view $\cdot$ Perspective $\cdot$ Perspectivism $\cdot$ Van Fraassen $\cdot$ COVID-19 $\cdot$ SARS-CoV-2 $\cdot$ Prescriptive practice $\cdot$ Built environment · Public engagement

This article belongs to the Topical Collection: Perspectivism in science: metaphysical and epistemological reflections

Guest Editor: Michela Massimi

Vadim Keyser

vkeyser@csufresno.edu

Hannah Howland

hhowland@pyatok.com

1 California State University, Fresno, California, USA

2 Pyatok, Oakland, California, USA 


\section{Introduction}

Scientific engagement by making measurements can be analyzed in terms of physical interaction with the world as well as information that is gathered from that interaction (van Fraassen 2008, 91). When asking questions like - what is the replication rate of SARS-CoV-2? - we are combining questions about the physical interactions, manipulations, and productions within the measurement process with questions about representation in information-gathering. That is, to address such questions we must focus on key interactions in the total measurement process, such as virus-to-host and built environment-to-social process interactions, in addition to how to selectively represent those interactions. A challenge for a robust philosophical account of measurement is to explicitly answer: What does the physical interaction of measurement consist of? What sort of representational information about the target of measurement is generated during the measurement interaction? Moreover, what are the relations between interaction and information-gathering?

Van Fraassen (2008) details the roles of representation ${ }^{1}$ and perspective ${ }^{2}$ in a thorough account of measurement practice. His account maintains the representational aspect of measurement theory as well as its focus on applied measurement practice, while loosening the strict mathematical standards of correspondence rules. The broad scope of his account applies, not only to 'representational' measurement practice, but also to 'productive' measurements. Additionally, he provides measurement methodology that is applicable to the empirical sciences. These components make his view comprehensive, dynamic, and flexible in its application to various scientific practices.

Our discussion takes inspiration from van Fraassen's (2008) account-specifically, the technical suggestions for two desiderata: information-gathering and physical interaction in measurement practice, which will serve as a starting structure for our account of the measurement process. Given his comprehensive focus, we believe that

\footnotetext{
${ }^{1}$ Early accounts of measurement focus on the systematic assignment of quantitative values (e.g. numbers or vectors) to objects in the world (see Helmholtz 1887; Campbell 1920). Nagel (1930) characterizes this approach to measurement as "the correlation of numbers with entities which are not numbers" (7). These early accounts approach the assignment of numbers to objects by looking at the conditions that make number assignment possible - e.g., by looking at the relation between numbers, scales, and magnitudes. This technical area of measurement theory has as its focus the mathematical representability of the physical world. The tradition of measurement theory is later continued by Stevens 1946; Suppes and Zines 1963; Ellis 1966; Pfanzagl 1968; Krantz 1964; Roberts 1979; Berka 1983; Kyburg 1984; and Narens 1985-among others; and is referred to, by philosophers of science, as the 'Representation Theory of Measurement'. Many problems arise for the Representation Theory of Measurement (Luce and Narens 1994). The problems do not imply that representation is the wrong approach to a theory of measurement, but rather that number assignment (e.g., physical-to-mathematical correspondence rules) may be the wrong way to express representation in measurement.

${ }^{2}$ It is important to note that Giere (2006) develops a similar account of the role of perspective in scientific modeling and observation. We refer to components of Giere's view that are relevant to the discussion. Other views, e.g., Teller (2018), Massimi and McCoy (2018), and Tal (2013) discuss important aspects of perspective and representation in measurement. The purpose of our discussion is not an organization of perspectival measurement accounts, but rather, an extension of perspectival measurement for disagreeing, complex, and developing measurement processes in the context of limited theory. Our will only focus on relevant philosophical accounts.
} 
van Fraassen's (2008) account provides the starting steps for a robust methodological account of perspectival measurement. We extend the account to be able to embed empirical practice where there is complex intervention, disagreeing results, and limited theory. It is our view that a robust measurement account can explicitly organize complex and developing measurement processes; but it can also make methodological suggestions for applied scientific practice. That is, such an account can serve as a model that embeds measurement practice by, e.g., explicitly representing important details in a given measurement processes. Such a robust account can also push measurement practice to explore new avenues of representation and production.

The specific aim of our account is to focus on the interactive side of measurement practice to provide a methodological prescription for developing measurement processes in the context of limited theory. We explicate the methodological importance of measurement interaction for information-gathering-specifically, theory development and multi-perspective measurement. Ultimately, our perspectival account of measurement will provide a methodological blueprint for organizing complex and developing measurement processes. The account should be useful to philosophers of science, who are interested in the intersection between representation and intervention; scientists, who are interested in methodological suggestions for theory-development and reliability; and interdisciplinary researchers, who are interested in the intersections between the pandemic, built environments, and social processes. As such, the purpose of this account is twofold: to present a working model of measurement in the context of developing measurement processes; and to guide applied measurement practice.

This discussion is outlined as follows. In Section 2, we structure van Fraassen's (2008) account, using four features that will be the building blocks for a robust account of perspectival measurement: Physical Interaction; Theoretical Characterization; Representational Content; and Perspectival Information. In Section 3, we extend the account to empirical contexts with complex intervention, disagreeing results, and limited theory. In Section 4, we apply the perspectival account to various aspects of measurement in the SARS-CoV-2/COVID-19 pandemic. In this section, we show how our account of perspectival measurement explicitly tracks key elements - manipulation/intervention, independence, invariance, and theory-development—by organizing unfolding measurement processes; and we also show that the account is useful for making methodological suggestions for unfolding measurement practices in SARS-CoV-2/COVID-19. Specifically, we discuss that many interactions in measurement processes, that are causally relevant for SARS-CoV-2/COVID-19 measurement outcomes, have not been properly acknowledged.

\section{Perspective in measurement}

Van Fraassen's (2008) provides a philosophical account of measurement that details the co-stabilization between theory and measurement practice. To analyze his account, we divide the discussion into three brief parts. First, we analyze van Fraassen's treatment of the views "from above" and "from within" scientific practice, which inform how to identify what is measured. Second, we discuss interaction and information-gathering in 
van Fraassen's account of measurement. Finally, we structure van Fraassen's account, using four features: Physical Interaction; Theoretical Characterization; Representational Content; and Perspectival Information.

\subsection{Views "from within" and "from above"}

According to van Fraassen (2008), we can philosophically account for a given measurement practice from two vantage points: 1) "From within" the historical development of theory and practice; and 2) "From above", that is, from the vantage point of a developed and established theory. The function of these views is to solve van Fraassen's reconceived 'problem of coordination'. 3

Van Fraassen summarizes the focus of this problem: how do measurements establish a value for what is measured $(2008,121)$ ? He uses Mach's definition of "coordination" to designate the process of assigning a value or location (e.g., a given thermometer value) to a given state (e.g., a state of heat) (van Fraassen 2008, 116). According to van Fraassen, Reichenbach (1965) specifies the 'problem of coordination' as a problem of "linking" mathematical structures to physical structures $(2008,118)$. Briefly summarizing Reichenbach's (1965) view on coordination is helpful in developing van Fraassen's version. In order to coordinate between two objects, the following conditions must be met: (a) the elements of each object are defined, and (b) there is a correspondence (usually in the form of a function) between elements in the set of the first object and elements in the set of the other object. Coordinating between an abstract mathematical structure and physical "reality" proves to be a problem according to Reichenbach. He says, "Although the equations, that is, the conceptual side of the coordination, are uniquely defined, the "real" is not. On the contrary, the "real" is defined by coordination to the equations" (1965, 37-38). Van Fraassen specifies the problem: How are "physical correlates", e.g., a light ray path as a correlate for geodesics, "to be identified without the use of geometric or kinematic terms" (2008, 119). He says that a mathematical structure can be coordinated with a concrete physical object "only in a context where something is already given that will make that possible" (van Fraassen 2008, 120-121). For Reichenbach this possibility is afforded by 'coordinating principles' and, later, 'coordinative definitions' (2008, 120). But van Fraassen argues that coordination is made "from within" the historical context of a developing scientific practice and then evaluated in retrospect, or "from above", from an established theory. To attempt to understand coordination from a "view from nowhere" creates an impossible problem (van Fraassen 2008, 122). Next, we discuss each view to show how it accounts for what is measured.

A view "from within" depicts the historically-located process of measurement where measurement procedures, including instruments and scales, co-stabilize with theory in order to characterize what is measured. For van Fraassen, the view "from within" a given measurement practice requires that we look "....into the introduction of measurement procedures at historical stages where there were already measuring procedures for certain other physical magnitudes taken as given" (2008, 122). We interpret the

\footnotetext{
${ }^{3}$ The purpose of discussing the revised problem of coordination is to motivate and explicate van Fraassen's co-stabilization between theory and practice. The discussion is not meant as a thorough analysis of evolution of the problem of coordination.
} 
general form of this solution to be: When measurement procedures become stable and a mature, theory emerges that can account for the stability of those procedures, and that theory identifies what is measured.

In van Fraassen's recreation of a view "from within" thermometry, there is a "joint evolution" between theory and practice: The air and liquid thermometers (and their theoretical details) are replaced by the gas thermometer and the empirical regularity expressed in Boyle's gas law, along with the theoretical characterization provided by kinetic theory. The end result of the joint evolution is that the gas thermometer and Boyle's gas law, along with kinetic theory, provide a characterization of temperature. The determination of what van Fraassen (2008) calls the "measurable quantity" (i.e. the "identified parameter") was achieved in many small steps where: 1) many theoretical assumptions were discarded (e.g., that all liquids behave the same when heated); 2) scales of measurement were replaced (e.g., ordinal with interval with ratio); and 3) the kinetic theory "finally" identified what was being measured.

Once theory and practice have stabilized, a view "from above" provides a theoretical description of what is measured, often leaving out or idealizing the historical evolution of measurement procedures. The next step is to structure van Fraassen's account of measurement by specifying his views on interaction and information in measurement, which contain elements from both the view "from above" and the view "from within".

\subsection{Interaction and information-gathering in measurement}

For van Fraassen, measurement interaction between an object of measurement and apparatus generates a physical outcome - the "measurement outcome" or "physical correlate of the measurement outcome" - which provides information content about the target of measurement $(2008,143)$. He says, "Measurement always involves a physical interaction between 'object' and 'apparatus'; that interaction we can call the physical correlate of the measurement" (2008, 143). The contents of measurement outcomes convey information about what is measured through the mediation of theory. Van Fraassen posits that theoretical characterization of measurement interaction requires 'coherence' between the theoretical characterization and measurement outcomes and their relation to what is measured $(2008,145)$. In short, the theory tells a coherence story about "how its outcomes provide information about what is being measured" (2008, 145). Furthermore, the information content is representational. Van Fraassen says, "The outcome provides a representation of the measured item, but also represents it $a s$ thus or so" $(2008,180)$. To understand how the representational relation works, it is important to refer to the 'representation criterion', which stipulates that the outcome of measurement ('appearances') selectively resembles the target of measurement ('phenomena') (2008, 141). For van Fraassen, phenomena are observable objects, events, and processes-whether observed or not $(2008,283,307)$. The outcome of a given measurement provides a perspective on a phenomenon - meaning that the content of measurement tells us what things look like, not what they are like (2008, $176,182)$. The content of the measurement outcome is an appearance, which is public/ intersubjectively-accessible and repeatable $(2008,276,284)$. An important qualification is that for van Fraassen, a representation does not represent on its own. The scientist selects the aspects/respects and degrees to which a representation represents a target. This relation can be expressed as: $\mathrm{Z}$ uses $\mathrm{X}$ to represent $\mathrm{Y}$ as $\mathrm{F}$, for purposes $\mathrm{P}$. 
Now that the target and outcome of measurement have been characterized, we can specify van Fraassen's role of theory in the act/operation of measurement. According to van Fraassen, "Measurement is an operation that locates an item (already classified as in the domain of a given theory) in a logical space, provided by the theory to represent a range of possible states or characteristics of such items $(2008,164)$. Three things are worth noting about van Fraassen's discussion of logical spaces. First, a logical space provides a multidimensional mathematical space that locates potential objects of measurement $(2008,164)$. By measuring we assign the item a location in a logical space. However, according to van Fraassen, it does not have to be on a real number continuum. As van Fraassen points out, items may be classified (by theory) on a range that is "an algebra", "lattice", or a "rudimentary poset" $(2008,172)$. Second, theoretical location depends on a "family of models" and not just an individual model $(2008,164)$. Third, an item is located in a "region" of logical space rather than at an exact point $(2008,165)$. If we take a view "from above", theory provides a classificatory system for what is measured. Importantly, theory is necessary for this type of classification. Van Fraassen says, "A claim of the form "This is an X-measurement of quantity $M$ pertaining to $\mathrm{S}$ " makes sense only in a context where the object measured is already classified as a system characterized by quantity M" (2008, 144 our emphasis). Additionally, for van Fraassen, measurement classification changes "in content and in truth conditions" as accepted theories change $(2008,143)$. We can infer that not only do parameters of physical systems change along with theory, but also whether a given measurement is "accurate" (2008, 139).

Summarizing van Fraassen's comprehensive account, produces four features for a developing perspectival account of measurement, which we call the Perspectival View of Measurement (PVM):

i. Physical interaction The interaction between apparatus an object produces a physical correlate of the measurement outcome.

li. Theoretical characterization The content of the measurement outcome is given a location in a logical space, which is governed by a family of theoretical models. An item's location within a logical space can change in content and truth conditions as accepted theories change.

lii. Representational content The content of a measurement outcome provides a selective representation of a given target of measurement (phenomenon). Because representations do not represent on their own, users and pragmatic considerations set the representational relation such that: $\mathrm{Z}$ uses $\mathrm{X}$ to represent $\mathrm{Y}$ as $\mathrm{F}$, for purposes $\mathrm{P}$.

Iv. Perspectival information Measurement generates appearances, which are public, intersubjective, contents of measurement outcomes. Appearances provide selective information about phenomena. Thus information from measurement tells us what something looks like and not what something is like.

Van Fraassen's (2008) discussion of interaction and information in measurement offers an intricate and fruitful account for analyzing scientific practice. It grounds a certain kind of objectivity because the phenomena are presumed to remain stable, even if our theories and practices may vary in successfully representing those phenomena. It 
also accounts for the limitations of our scientific practices by emphasizing that we produce selective representations in our measurement outcomes. However, our aim is to extend information gathering and interaction to disagreeing, complicated, and developing cases of the measurement process. We will, first, address interaction in the act and process of measurement; and then we will turn to the treatment of information gathering. It is important to note that these two problems are not necessarily related, even though in our discussion, they build off of each other. That is, to solve one does not imply solving the other. However, both must be addressed to adequately account for measurement interaction and information.

\section{Interaction, manipulation, and production in the measurement process}

In this section we extend PVM to address the relationship between interaction and information-gathering in the measurement process. How can the features of PVM, developed so far, account for measurement practice, where measurement conditions must be defined beyond instrument and object distinctions? How can PVM account for measurement practice without the presence of theory; or, no theory and multiple measurement practices? In this section, we focus on the methodological importance of measurement interaction for information-gathering. But the larger aim is to test drive PVM on multiple terrains of scientific practice in order to see where it breaks in accounting for scientific practice; and, in the next section, to see where it fails/ succeeds in making methodological suggestions for the uncertain measurement context of a sociobiological pandemic. In this section, we focus on the methodological importance of measurement interaction for information-gathering.

\subsection{A process-view of measurement interaction}

To comprehensively explicate measurement practice, an adequate account of measurement should be able to track the total process of measurement interactions, not just the act of measurement, in order to describe how measurement manipulation/intervention produces the outcome of measurement. We argue for a process-view of perspectival measurement that explicitly embeds both productive and representational roles in the measurement process. In this section, we outline a new feature of PVM, one that provides a useful set of relations to organize information and interaction in the measurement process. We show that the measurement process not only consists of the final measurement reading (or outcome), but it also consists of the manipulation of conditions in relation to specific scientific purposes. We begin with the issue of measurement interaction: What are the interactions involved in the total measurement process?

Van Fraassen emphasizes the representational role of measurement. The instrument produces an outcome that stands in a representational relation to the thing measured (2008, 374 our emphasis). We can summarize this, using the combination of physical interaction and representational content: The interaction between apparatus and object produces a physical correlate of the measurement outcome, the content of which provides a selective representation of a given target of measurement. But he also 
develops a non-representational role of measurement. With reference to Heidelberger (2003), Van Fraassen argues that instruments can function as "engines of creation"with a functional role to produce new observable phenomena. Regularities can be controlled in order to produce a new thing that is instructive about nature $(2008,95-$ 96). Van Fraassen's specifies that language of "production" is more appropriate than language of "discovery", although there is somewhat of a discovery when new things are produced $(2008,95)$. We can generate a general philosophical description: an engine of creation, or a productive measurement, is determined by measurement conditions that consistently create a given regularity.

For the purpose of developing PVM, we can be more explicit about how these conditions figure in the total process of measurement. This explication will have as its focus the selective manipulation of measurement conditions. We also extend the role of manipulation to more than just instrumentation. There are two reasons for this: 1) Measurement in the non-physical sciences offers complex and sometimes blurred relations between instrument and object of measurement; 2) Measurement is not merely the act of measurement. We briefly describe (1) and (2).

On van Fraassen's characterizations of measurement, the role of the instrument/ apparatus seems to have an important, if not necessary, mediating function. It may be the case that van Fraassen focuses on case studies (e.g., thermometry, microscopy, time measurement, and quantum measurement) that are instrument-intensive; and that his view of measurement is not instrument-centric. Whether or not the necessary mediating role of instruments is an explicit part of van Fraassen's account, there is room to develop a philosophical view of the measurement process. Without such philosophical development, we risk mischaracterizing complex cases of measurement. For example, there are cases of measurement where the distinction between apparatus and object measured is not as clean-cut as it is in cases of e.g., thermometry. By focusing on just the apparatus and object distinction our philosophical theory of measurement misses all of the important ways in which measurement conditions are manipulated in the measurement process: Scientists re-arrange biotic and abiotic measurement set-ups to expose organisms to various conditions which influence the development of those same organisms (Prasolova et al. 2006). Model organisms are standardized to measure chemical effects (sometimes by engineering them with partially human organs). Organic matter can even sometimes function as an instrument-e.g., the FourU thermometers, which are RNA molecules that act as thermometers in Salmonella (Waldminghaus et al. 2007).

Additionally, the process of measurement is not only about taking measurementse.g., getting a measurement outcome. That is, even in cases where there is a sharp apparatus-object distinction, the philosophical focus should not merely be on the representational content within the measurement outcome. Without developing a broader view of the measurement process, we risk conflating the act of measurement - e.g., locating a measurement outcome in a logical space-with the process of measurement. The act of measurement can be something as simple as putting a thermometer up to an object. But the process of measurement requires manipulating, re-positioning, and arranging causal conditions relevant to a phenomenon. ${ }^{4}$ As

\footnotetext{
${ }^{4}$ This characterization will be particularly important in Section 4.2, where we discuss how the pandemic rearranges causal conditions.
} 
discussed in Section 2, measurement is selective. However, this type of representational selection accounts only for the output of measurement. There is also selectiveness in the process of measurement. This can be stated as a new feature of PVM:

Selective manipulation In the process of measurement, scientists selectively manipulate certain causal conditions for specific scientific purposes - such as for the purpose of generating repeatable, reproducible results.

Before illustrating selective manipulation by using an empirical example, it is important to characterize 'measurement process'. This will provide a fine-grained view of how physical interaction works in measurement. We base our use of 'measurement process' on certain definitions from the International Vocabulary of Metrology - Basic and General Concepts and Associated Terms (VIM), 3rd edition (JCGM 2012). VIM uses 'measuring system' to indicate a single instrument or assembly of instruments that provide information about a system (JCGM 2012, 3.2). We use 'measurement process' in a broader way than VIM's 'measuring system' to encompass the following important aspects of measurement:

Preparatory activities Different types of preparation may occur prior to obtaining a specific measurement reading. In microscopy, for example, scientists prepare samples to meet the standards of the given microscope. Kinds of preparation are dictated by the specifics of each science.

Measurement conditions The variation and manipulation of conditions within a measurement setup may extend beyond instrumental manipulation. For example, variables can be manipulated prior to taking instrumental readings. As we discuss in Section 4, relevant conditions for SARS-CoV-2 measurement extend beyond the lab. ${ }^{5}$

Measurement procedures. Detailed descriptions (JCGM 2012, 2.6) of the different levels of measurement are interwoven with the measurement practice. This information may include measurement principles and methods (JCGM 2012, 2.5, 2.9).

The measurement result (JCGM 2012, 2.9) is not the only aspect of the measurement process worth noting. Important interaction occurs during preparation and measurement condition manipulation. But in VIM, there is a warning about measurement interaction:

The measurement, including the measuring system and the conditions under which the measurement is carried out, might change the phenomenon, body, or substance such that the quantity being measured may differ from the measurand as defined. In this case, adequate correction is necessary. (JCGM 2012, 2.3 Note 3, our emphasis)

Measurement interaction can create discrepancies that problematize precision and accuracy-hence the need for measurement correction. But measurement interaction can also produce changes to a given phenomenon, resulting in methodological use for

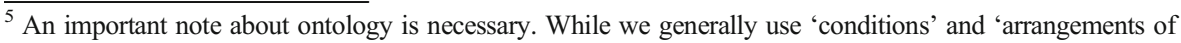
conditions' to refer to aspects of the measurement process, a 'process' ontology is often just as fitting. Our discussion focuses on methodological characterizations of measurement processes - where sometimes parameterization based on conditions is important for a given scientific purpose; and other times a focus on processes and process intersections is important for other purposes.
} 
generating repeatable, reproducible objects of measurement. To see how this works, we use the example of measurement in thermometry.

In the history of thermometry, fixed points, e.g., boiling point of water, served as a standard for calibrating different thermometers. One might think, "When scientists measure boiling point they merely take a reading with a thermometer." But there is also productive measurement. Briefly, we use Hasok Chang's (2004) discussion manufacturing fixed points in order to illustrate how measurement conditions can be selectively manipulated for specific measurement purposes.

When sorting through the history of fixed points, it becomes apparent that the point at which water boils depends on the manipulation of material conditions within the measurement process. Initially, it was discovered that boiling point varies with differences in atmospheric pressure (Chang 2004, 15). Additionally, the presence of dissolved air in water produced ebullition-like phenomena at 101.9 degrees $\mathrm{C}$ (Chang 2004, 19). However, purged water (water without dissolved air) was measured to behave in a phenomenologically similar manner at much higher temperatures (as high as 140 degrees C). According to Chang, scientists began to focus on samples of water without dissolved air (2004, 16-19). Chang presents an anecdote about how De Luc walked, slept, and ate for 4 weeks straight — all while shaking a tube of water to purge it of the dissolved air. De Luc's dedication to manipulating the conditions of measurement serves as a good illustration of the care with which the conditions of measurement have to be chosen and then manipulated. It also illustrates how sensitive measurement results can be to the interaction between measurement conditions. There are numerous other experimental conditions that show a clear demarcation in phenomena. For example, Gay-Lussac demonstrated that water boiled at different temperatures when boiled in a glass vessel vs. in a metallic vessel. Throwing some finely powdered glass into the glass vessel brought the temperature of the boiling water down; and throwing iron filings brought it down even more. So when measuring a boiling point, what measurement conditions were selectively manipulated (e.g. atmospheric pressure, presence of dissolved air, kind of vessel used, presence of other material in the sample, etc.); and how did those conditions work together?

The next step is to discuss the relevant scientific purpose for selectively manipulating the conditions. By carefully controlling these measurement conditions we can reproduce the phenomenon. For pragmatic purposes we would have a hard time keeping track of these different conditions and different phenomena (e.g., purified water boils at temperature $\mathrm{T}$, when it is boiled in a glass tube with distance $\mathrm{D}$ between the thermometer and the bottom and atmospheric pressure A, and so on). Pragmatically, we need a stable phenomenon in order to establish thermometry as a successful enterprise. As the story of boiling point unfolded, the conditions for the boiling point of water were stabilized. It was not only that scientists were stabilizing our concept of 'boiling point', but it was that they were stabilizing the material conditions so that they could have repeatable, reproducible results. ${ }^{6}$

From this example we can conclude that atmospheric pressure and dissolved gas were selectively manipulated for the scientific purpose of having repeatable,

\footnotetext{
${ }^{6}$ We come back to the concept of 'stabilization' in Section 4.2, where we discuss destabilization by SARSCoV-2.
} 
reproducible results. ${ }^{7}$ In a fully developed measurement practice we may abstract out the specific steps in manipulating certain measurement conditions, thereby losing important causal details when describing the measurement process. If the measurement practice is stable enough, this may not matter for scientific purposes. But in developing a philosophical account of measurement interaction, we should strive to understand important features of the measurement process. Selective manipulation provides a useful set of relations to organize information about the measurement process. That is, the measurement process not only consists of the final reading, but it also consists of the manipulation of conditions in relation to specific scientific purposes. It is important to note that although 'manipulation' refers to causal interaction, it does not depend on a particular view of causation. In fact, the view of causation may change when the condition is applied to specific sciences (e.g., quantum mechanics vs. ecological measurement). But selective manipulation provides a way to track information in the measurement process. For instance, how can we track SARS-CoV-2 replication rate by manipulating conditions in measurement processes?

\subsection{Tracking information in developing measurement processes}

Ultimately, PVM provides a methodological blueprint for organizing complex and developing measurement processes. In this section, we present a new feature for PVM that addresses how developing and disagreeing measurement processes can be resolved through theory-modification. We specify that selective manipulation of causal conditions can lead to the modification of assumptions that characterize measurements, and eventually to modification of theory and resolution of disagreeing results. Simply put, we outline how disagreeing results can prompt a methodological process, which culminates in theory-building and the resolution of those disagreeing results.

As van Fraassen says, measurements can distort given that instruments have a limited range $(2008,183)$. While van Fraassen does not provide an explicit account of 'accuracy', his use of the term offers an important suggestion: specifically that judgments about accuracy require established theory. ${ }^{8}$ According to van Fraassen, "Within the vantage point of the accepted theory, once such stability has been achieved, we can speak meaningfully of the accuracy with which a given instrument gauges a given observable" (2008, 139 our emphasis). On this view, the methodological backbone of accuracy judgments seems to be co-stabilization between theory and practice. Importantly, this evaluation of accuracy requires a well-developed theory, which is

\footnotetext{
$\overline{7}$ This scientific purpose may be generalizable to many sciences, and for this reason may be a key component in 'selective manipulation'.

${ }^{8}$ Our discussion will be about the initial steps of theory-modification, and characterization that occurs prior to more developed judgments about accuracy. For further discussions about 'accuracy', see Tal (2013) and Teller (2018), in addition to International Vocabulary of Metrology - Basic and General Concepts and Associated Terms (VIM), and the Evaluation of Measurement Data - Guide to the Expression of Uncertainty in Measurement (GUM). We take VIM's qualified methodological definition to be particularly important for evaluating accuracy judgments in the context of well-developed theory. Measurement accuracy is used to define closeness of agreement between a measured quantity value and a true quantity value (or, set of quantity values) of a measurand (JCGM 2012, 2.13). The qualification of the 'Uncertainty Approach' is important: Due to the inherent incompleteness in the detail of the definition of a quantity, there is a set of true quantity values, and this set is in principle and in practice, unknowable (JCGM 2012, 2.11 Note 1). Because of an incomplete definition of the measurand, the measurand has "intrinsic" uncertainty.
} 
characteristic of a view "from above" discussed in Section 2. However, there are areas of science with developing theory-e.g., ones with conflicting measurement results and, seemingly, no way to make sense of the results. Before we can achieve anything close to measurement accuracy, the initial step is how to build theory and to characterize measurement results.

It is important that PVM is adequate in the context of developing scientific practice. An example of measurement from pheromone research illustrates the point. We suggest that there needs to be a methodological supplement to characterize measurement processes and results in the context of developing theory. In Doty's (2010) comprehensive account of the existence of pheromone effects in humans and animals, there are two conflicting measurement perspectives and results. We will refer to these as 'the behavioral measurement perspective' ('BP' hereafter) and 'the neurophysiological measurement perspective' ('NP' hereafter). The two types of perspectives use different physical processes and assumptions, and they disagree.

Doty (2010) reports the influence of pheromones on many different factors of human behavior - such as, autonomic nervous system function, social response, and the evaluation of others $(2010,149-153)$. All of these results have something in common: Subjects are exposed to a chemical pheromone agent and a behavioral effect is observed. For example, androstenone and androstenol are causal conditions that can be manipulated within a given measurement setup (e.g., in the context of seating choices in a waiting room) and a specific effect is observed. There is no physiological measure on specific chemical pathways. Importantly, BP contains many assumptionse.g., subjects are not under placebo or nocebo effects, pheromone-induced behaviors can be differentiated from other behavioral effects due to e.g. aesthetic judgments about odor, pheromone behavior responses are universal and consistent within a given sample, etc. While different studies use different pieces of equipment it is important to note that many studies share the same type of samples (e.g, androstenol from the same manufacturing source), and many of the studies share the same procedures, which dictate how instruments and conditions are to be manipulated. For example, Doty (2010) cites pheromone studies that have over twenty replications with little change in the procedure.

The neurophysiological approach provides a sharp contrast in methods and results. According to the results generated by neurophysiological measurement, pheromones do not influence human behavior. Using specific neural measures, it was discovered that the adult human vomeronasal organ (the organ responsible for processing pheromones in other animals) lacks the VNO-specific TRP2 membrane channel, a nonselective cation channel critical for VNO function in other animals. Additionally, the VNO in humans has no centrally projecting neural connection. Finally, humans lack the brain structure to which vomeronasal nerves ordinarily project $(2010,130)$. Each of the results points to the non-functionality of the human VNO. The key assumption is that the VNO is necessary for pheromone communication; and because it is non-functional in humans, we cannot process pheromones. According to the neurophysiological approach, since humans cannot process pheromones, the behavioral approach results are in error.

This juxtaposition between BP and NP offers a puzzle for pheromone measurement. Each of the research approaches contains results that converge with other results from that same approach. But between-approaches comparison shows 
disagreement in results. Moreover, BP has dozens of studies in support, while NP only has a few. Are pheromone effects in humans genuine as BP suggests, or are they the result of error(s) as NP suggests? Without well-developed theory, judgments about accuracy are absent. However, the comparison between disagreeing measurement results can push theory development. That is, in the absence of theory, scientists can still selectively manipulate conditions to test both the behavioral and neurophysiological aspects.

In one study (Dorries et al. 1997), researchers manipulated the VNO in domestic pig (Sus scrofa) to observe its role in pheromone communication. Chemical access to the VNO was blocked using surgical cement. We can structure the predictions and results of this study in order to make interesting philosophical conclusions about the interaction between theory and results. The predictions and consequences are as follows. If the VNO is necessary for pheromone communication then there should be no behavioral effect; and the results will converge with NP. If the VNO is not necessary, then there should be a behavioral effect; and the results will converge with BP. The actual result from the experiment converged with BP (Dorries et al. 1997). That is, pigs were as responsive to pheromones with the blocked VNO function as they were with regular VNO function. The consequence of these results is that NP runs on an improper assumption about the necessity of the VNO. Once this assumption is modified and we discard NP, results no longer disagree. But it is the disagreement that prompted this methodological resolution in the first place! The next step is for scientists on the theory side of pheromones to explore new mechanisms for pheromone communication-such as, absorption through the lungs and stomach lining.

We can abstractly organize the measurement methodology in order to develop an important feature of PVM. In the pheromones case study, manipulation is methodologically useful because it provides systematic tracking of measurement conditions and results - e.g., if we temporarily block the VNO processing, will results diverge from BP? The manipulation dictates theoretical modification: Results of the new experiment prompt the elimination of the problematic assumptions about the necessity of the VNO. The assumptions have a mediating role in theory modification. In Section 2, we presented theoretical characterization, which outlines that theory is used to characterize what is measured. But in the absence of theory, assumptions can provide a partial characterization of what is measured. The characterization of variables and relations is a work-in-progress-i.e. for the purpose of producing further measurements. It will vary on a case-by-case basis at what point the arrangements of assumptions become theoretical models. This is an issue for a later time. For now it is important to highlight the methodological importance of manipulation in theory-modification:

Theory-modification: In context of disagreeing measurement processes, scientists can selectively manipulate certain causal conditions for the purpose of modifying assumptions that are the backbone for characterizing what is measured.

A philosophically interesting interaction between theory and practice emerges: Disagreement in results is characterized by theory but it can also prompt specific theory-modification. Moreover, the role of multiple disagreeing measurement perspectives is essential. Measurement results from many perspectives push the details of theory. So far, PVM has explicitly addressed the role of manipulation for the measurement process (interaction) and for theory-modification (information). But the role of 
many perspectives or processes needs philosophical attention in order to address nuanced questions like: Can any disagreeing measurement perspectives be useful for theory-modification? The final step in the development of PVM is to explicitly address how to combine multiple perspectives or multiple measurement processes to yield useful information.

\subsection{Tracking information in multi-measurement processes}

In this section, we further develop the methodological importance of measurement interaction for information-gathering. Specifically, we explicate a relationship between selective manipulation and multi-measurement process convergence and divergence. We discuss two methodological points about measurement in the context of limited theory. First, manipulation provides a method for eliminating dependent relations between multiple measurement processes. Second, there is a methodological value to disagreeing (divergent) results in the presence of manipulation. Both of these points complement the aforementioned feature of PVM, theory-modification via manipulation of conditions.

In the context of multiple developing measurement processes, can results be methodologically useful for theory-modification? The answer is that it depends on the type of multi-perspective agreement (convergence) and disagreement (divergence); moreover, it depends on the role of manipulation in generating convergence or divergence. In this section, we provide a distinction between two types of information that are provided in measurement. The first is invariant information, generated from convergent measurement results. The second is varying information, generated from divergent measurement results. Broadly, invariant information remains steady with admissible changes in measurement processes. ${ }^{9}$ Varying information is sensitive to changes in measurement processes. Both types of information are also useful for understanding how measurement interaction is informative in the context of multiple perspectives. For example, if a given instrument's readings vary under a specific set of conditions, we learn something about the sensitivity range of that instrument. If a specific measurement production arises in the context of multiple independent ways of detecting it, we can conclude that it is unlikely that it is produced by a systematic error. As shown by the pheromone example, even in the absence of developed theory, information can be usefully tracked, and assumptions modified, when multiple incompatible perspectives are involved. But invariant information vs. variant information across perspectives needs to be further specified to be methodologically useful.

Giere (2006) makes an instructive point, broadly applicable to overlap between perspectives. Sometimes instruments have overlapping or invariant perspectives. Giere says, "Is this [overlap] not good evidence that there is something "objectively" there? Indeed, this is good evidence that there is something there, but need not be understood as knowledge in an "absolute objectivist" sense" (Giere 2006,

\footnotetext{
${ }^{9}$ For van Fraassen, invariance seems to play two methodologically important roles. First, it indicates what is not a "real thing" $(2008,102)$. Second, it helps to "correctly and accurately ascertain" something "in certain respects" (2008, 73). Importantly, van Fraassen's use of 'invariance' applies to ascertaining spatial structures in certain respects (van Fraassen 2008, 73). We broaden the use of 'invariance' to more than structural analysis, while fine-tuning it for methodological use.
} 
58). The reason why we cannot make "absolute objectivist" claims is because multiplying perspective does not give us a perspective-less view. Giere adds, "The simple and fundamental point is that to be an object detected in several different perspectives is not to be detected in no perspective whatsoever" $(2006,58)$. Using Giere's example of the speed of light, we extend the analysis. Consider the speed of light, $c$, which figures centrally in the content of different theoretical perspectives. Giere says that $c$ 's invariance over many perspectives does not mean that it is independent of any perspective. Adding to Giere's point, by looking at the invariance of $c$ over different perspectives, we cannot distinguish between two options:

1) The speed of light is a measurement result that is independently-generated by each perspective (or each measurement process). (Here we use 'independence' in a minimal way to indicate that a result in each measurement process is not influenced by the results of other measurement processes.)

2) The speed of light is a posited, idealized theoretical quantity which helps to make sense (mathematically, conceptually, etc.) of dependent perspectives, each relying on the other(s) for support.

Giere's point is that the perspective-independence of some phenomenon ${ }^{10}$ cannot be inferred from its repeatability in multiple perspectives. The further point is that the particular type of perspective dependence cannot be inferred from repeatability in multiple perspectives. In other words, repeatability across perspectives does not distinguish between independently-generated invariants (1) and perspective-dependent invariants (2). This point is important because it flips the epistemological lens on to the status of our measurement practice. For a given applied measurement practice this could be a devastating problem to address. If we cannot specify multi-perspective dependence of our result about SARS-CoV-2 transmission, then how can we infer that the result does not emerge from a systematic error across those perspectives? One might posit that to distinguish between independently-generated invariants (1) and perspective-dependent invariants (2), an account of perspective independence is necessary. There has been much discussion about the methodological and inferential benefits of an account of independence. ${ }^{11}$ To be relevant to this discussion, an account of 'independence' would have to apply to cases where there is limited or no theory. There are accounts of independence that are not theory-heavy. For instance, Woodward's 'measurement robustness' consists of using different measurement procedures to converge on the value of some quantity $(2006,234)$. Independent procedures “...involve instruments of different design, operating according to different causal principles, they employ different assumptions to interpret the data they produce and so on" (Woodward 2006, 234). But such characterizations of differences-e.g., in

\footnotetext{
${ }^{10}$ Giere uses 'real system' in a similar fashion to van Fraassen's 'phenomenon'.

${ }^{11}$ Philosophers have addressed the 'individuation' (independence) of modes of measurement and also evidence. For discussions on independence see Horwich (1982); Franklin (1997); Sober (1989); Trout (1998); Culp (1994); Keeley (2002); Staley (2004); Wimsatt (2007) and Stegenga (2009, 2012); Lloyd (2015) and Schupbach (2016). Much of this literature intersects with literature on 'robustness analysis'. We deviate from this literature in a particular epistemological respect. Even in the presence of independence, diverging results are characterized as results produced in error (Wimsatt). Divergence ('discordance') in results has also been argued to thwart useful inferences about evidence (Stegenga 2009). But we argue that there is methodological usefulness in disagreeing or divergent results.
} 
procedural operation and causal principle-would require well-developed theory. ${ }^{12}$ When theoretical characterizations are missing, is there something that can be methodologically performed to, at least, begin the process of differentiating measurements? Below, we outline how to characterize 'independence', methodologically, and why measurement interaction/manipulation figures centrally in the characterization. Distinguishing (1) and (2) proves to be as challenging as it is fruitful about measurement manipulation analysis.

Instead of giving necessary conditions for the physical independence of measurement processes, we specify 'independence' in terms of a methodological indicator for independence - a 'manipulation indicator'. This methodological indicator is compatible with other forms of evaluating independence in order to make stronger claims about independence in e.g., assumptions, physical conditions, causal processes, etc. The reason why this interaction-based view is helpful is because it does not rely on any particular story about the co-stabilization between theory and practice. It is purely methodological in the sense that it offers a useful heuristic for distinguishing measurement processes. It also does not rely on a "view from nowhere" on our measurement practice. We are operating in van Fraassen's "view from within" practice.

Suppose that there are two measurement processes, M1 and M2. To see if M1 and M2 are independent we can introduce a manipulation to the measurement conditions ${ }^{13}$ behind M1 to see if it produces a change in M2's results. ${ }^{14}$ If it does, then there is successful manipulation, and we conclude that M1 and M2 are not independent. So two measurement processes are indicated as independent just in case manipulations to one measurement process will not change the function of the other measurement process. There are two apparent criticisms. One is that clearly this cannot be an account of independence because to indicate that two measurement processes are truly independent, all possible manipulations to the measurement conditions must be performedmoreover, they must be performed in all possible circumstances. Otherwise, the indicator is that two measurement processes are not dependent based on a particular manipulation. The second criticism is that suppose that M1 and M2 pass the manipulation indicator, but they share the same theoretical or measurement assumption. Consequently, any benefit in independence would be compromised by e.g., dependent measurement interpretations. ${ }^{15}$ These two criticisms prompt an important qualification: The methodological heuristic for independence should be framed as indicating partial

\footnotetext{
${ }^{12}$ A similar point can be said about the metrological specification of measurement principles. A 'measurement principle' is the "phenomenon serving as the basis of measurement" (VIM 2012, 2.4). To specify or differentiate (multiple) measurement principles, well-developed theoretical characterization is necessary.

${ }^{13}$ These can be instrumental conditions, specimen preparation conditions, and/or background conditions. It can be argued that in order to differentiate conditions, a theory is necessary. But conditions can be differentiated using more basic characterizations - e.g., perceptual concepts for object differentiation, or more general methodological concepts about scientific practice.

${ }^{14}$ Here we borrow Woodward's (2003) operation of manipulating one variable to see changes in another. However, our account does not rely on a specific theory of causation. Woodward's (2006) specification of causal robustness is also useful. Causal robustness focuses on invariant relationships over interventions or manipulations in conditions. Taking this a step further, if we observe converging or diverging results after we have modified a given variable, we can specify under what conditions this combination of agreeing and disagreeing results occurs.

${ }^{15}$ Thank you to two thorough anonymous reviewers for these points. They highlight much work to be done on a methodological account of independence.
} 
independence. Moreover, in strict interpretation, it is an indicator that eliminates dependent relationships within a particular interaction or type of interaction. The process of eliminating dependent relationships is precisely what is useful for tracking information in measurement practice; and it is only possible through involved measurement interaction. Additionally, this methodological account can be combined with other accounts in order to robustly define independence. For instance, it can be combined with Woodward's (2006) characterization of independence in assumptions.

Suppose that a staining procedure on cellular samples compromises the independence of each observation of cellular structure because the procedure introduces cellular damage into each observation. ${ }^{16}$ In this case the particular type of staining procedure is the manipulation indicator. But if we were to further compare the different types of staining procedures, we learn two things. First, we learn that the damaged sample is produced only in certain measurement circumstances. Second, we learn exactly under what conditions the damaged sample is produced. That is, if each measurement process is indicated as independent, we can compare the measurement processes to have them "check each other's results"-i.e. to identify where certain features arise in specimen preparation conditions, and to begin to develop a theoretical explanation that applies to those features. This implies that it is not merely convergence that is useful for information, but rather the manipulations between measurement processes that allow us to track information. If the results of each process disagree then we can compare the processes to locate the differences in productions; and in the presence of a developing theory, we can locate where error is being produced. ${ }^{17}$ Likewise, if the results of each process agree, it indicates that the results are less likely to be due to a common manipulation ${ }^{18}$; and in the presence of a developing theory, this can become an inference about the elimination of some systematic error.

\footnotetext{
${ }^{16}$ In this case we do not even have to characterize it as "damage". We can frame it as a structural feature without detailed theoretical development. The mesosome provides a fascinating example of such a case study (Culp 1994; Rasmussen 2001; and Wimsatt 2007).

${ }^{17}$ Once theory is in stable enough form, error can be sought out, e.g., as characterized in VIM: "measured quantity value minus a reference quantity value" (JCGM 2012, 2.16). There is no need to put the current discussion into VIM terminology for reasons discussed in Section 3- e.g., the need for broader definitions of measurement processes and conditions. But if we entertain metrology, side-by-side with this discussion, an important issue emerges - is there a point before theory is developed "enough", where practice will not grant useful information tracking? The Evaluation of Measurement Data - Guide to the Expression of Uncertainty in Measurement (GUM) provides an important concern about lack of adequate measurand specification and discrepancies in results: "Inadequate specification of the measurand can lead to discrepancies between the results of measurements of ostensibly the same quantity carried out in different laboratories" (JCGM 2008, D.3.4. Note 3). It might be that multi-measurement practice, alone, provides inadequate specification of a measurand, and thus discrepancies in results that cannot be properly characterized. However, it is important to grant that specification of the measurand does not have to come from the theory that is currently being developed. Independently developed theoretical and measurement concepts can step in, just like the barometer aided in the development of the thermometer.

18 This is where in the presence of theory, we can get something closer to Woodward's measurement robustness procedure independence. According to Woodward, the convergence in measurement robustness increases our confidence that the quantity has been measured "accurately" because the independent procedures used are unlikely to have the same error $(2006,234)$.
} 
This methodological process amounts to a more modest claim than, invariant results indicate what is "real". The implication is that by manipulating and tracking multiple measurement processes, we signpost and identify features of those processes - that in the ongoing development of theory can be turned into the identification of error and accuracy. ${ }^{19}$ That is, by comparing conflicting processes we can usefully identify where error is being produced in the measurement process. But notice that this is an ambitious claim about what we can epistemologically infer about our measurement practices. By manipulating, we eliminate dependence-relations and make inferences about independence. Manipulation serves as a mechanism for mapping out the specific relations between measurement conditions within our measurement processes.

By explicating multi-measurement methodology we can also update the theorymodification feature in PVM:

Multi-process theory-modification: In context of multiple measurement processes, scientists can selectively manipulate certain causal conditions in each measurement process to analyze invariant and varying information for the purpose of modifying assumptions that are the backbone for characterizing variables.

This update sets the methodological blueprint for the relations between multiple measurement processes, manipulation, invariant and varying information, assumptions, and theory-modification. The next step for this account, for both philosophers of science and scientists, is to apply it to be informative about an unfolding measurement practice. While such a project is too large for this discussion, we make important initial steps for application to case studies like SARS-CoV-2 measurement.

\section{Pandemic: SARS-CoV-2 measurement organization; COVID-19 measurement prescription}

The purpose of this section is two-fold. It is to show that a detailed theory of measurement can serve as an organizational tool for a developing case study with complex measurement interactions; but it is also to show how a theory of measurement can make prescriptive suggestions for measurement practice. ${ }^{20}$

\subsection{SARS-CoV-2 measurement organization}

The features of PVM, can be selectively used to make important explications about unfolding measurement in SARS-CoV-2. One major issue is measuring how the virus behaves. This is where a methodological account of measurement can be useful. In vitro measurements are not enough to get a clear perspective of the complicated interactions involved in transmission and replication, so epidemiological, model organism, and computer simulation measures are being developed. Based on previouslydeveloped measurement perspectives about SARS-CoV and the likeness to SARS$\mathrm{CoV}-2$, scientists are piecing together a theoretical picture, filled with many

\footnotetext{
${ }^{19}$ We take judgments about 'error' and 'accuracy' to be theory-relative. This is also consistent with VIM's aforementioned definition of 'accuracy' where true quantity values and corrected values are made relative to measurement principles, which will ultimately require theoretical specification. In the absence of welldeveloped theory, low-level theory can also be employed to indicate some sort of operationalization.

20 This section was developed in March, 2020.
} 
representational paradoxes. For example, it is believed that ACE2 serves a main mediating role in the transmission of the virus, even though, paradoxically, blocking ACE2 does not stop transmission and may even make progression of pneumonia worse (Chen et al. 2020). Can a philosophical measurement theory help to explicitly organize the measurement process and the details of methodology, assumptions, and theory?

One point of focus for PVM is the total measurement process. That is, measurement is not just about taking a measurement - e.g., observing the replication rate of SARS$\mathrm{CoV}-2$ in vitro. Analyzing the total process of measurement, including complicated measurement interactions, may hold the key to adequately characterizing e.g., 'replication' in SARS-CoV-2. We present a small slice of SARS-CoV-2 measurement that can be dissected and organized using PVM. First, it is clear that the behavior of SARSCoV-2 - e.g., transmission and replication-depends on many factors, such as environmental conditions, surface structure/topography, and ACE2 profile, among other conditions. But such a list of conditions, merely provides a complicated puzzle with many pieces to tell a representation story. For example, given a certain temperature and immune profile, virus replication may be difficult to precisely represent. Theory requires development to characterize what is measured.

However, there is an even more fascinating explication of the measurement process: SARS-CoV-2 replication measurement is also productive. That is, depending on the causal condition interactions involved in the total process of measurement, replication rates can dynamically change. Specifically, virus-to-host interaction can change the virus genome, which can drastically alter replication. It is interesting to note that van Fraassen $(2008,95)$ concedes that scientific 'engines of creation' may also be realized in nature. We express this in a slightly different way, consistent with the discussion of manipulation and production in Section 3-the arrangement of conditions involved in productive measurements are not necessarily human-centric. That is, causal conditions that are relevant to the measurement process, can be altered by scientists, but they can also be altered by the complexity of interactions prior to or during the act of measurement. In SARS-CoV-2 measurement, nature has arranged some of the interactions - e.g., between host and virus; and it is precisely these arrangements that are relevant to the total process of measuring replication. Briefly, we show the start of the measurement story. We emphasize key parts of PVM that pertain to the complex interactions involved in the measurement process as well as independent and invariant measures. The takeaway is that PVM can be used to suggest a broad view of the complex interactions in the measurement process, and it can be used to reclassify measurement phenomena, like 'replication'.

According to $\mathrm{Su}$ et al. (2020), samples from hospitalized patients in Singapore showed an interesting virus-to-host interaction. Specifically, they found a 382-nt deletion that spanned nearly the entire 22 open reading frame 8 (ORF8) of SARSCoV-2. Additionally, Su et al. (2020) found deletions in ORF8 transcription-regulatory sequence. Without well-developed theory, this measurement result can be used to create an elementary assumption: SARS-CoV-2 viral genomes can be modified as a function of host interactions. It is an interesting assumption because it changes the location of SARS-CoV-2 within a methodological space. Understanding SARS-CoV-2 as a function of its productive conditions, it can be characterized as sensitive and unstable in the context of its host interactions. This can prompt further experimental designs that test virus-to-host manipulations. But alone, this methodological space 
location is not informative about SARS-CoV-2 replication. That is, how does understanding SARS-CoV-2-to-host interaction benefit any scientific purpose that aims to understand replication? This is where the methodology of measurement independence is helpful.

Independent measures of from Muth et al. (2018) have suggested that these deletions in ORF8 resulted in reduced replicative fitness of SARS-CoV. Unlike the $\mathrm{Su}$ et al. (2020) study, the measurement results were not representations from clinical sites. Rather, Muth et al. (2018) engineered full, partial, and deleted ORF8 into a SARS-CoV infectious cDNA clone, and then introduced this clone to various physiological systems, mediated by ACE2 receptors. These are productive independent measures that indicate the causal role of ORF8 in replication. That is, the measures are generated from the arrangement of causal conditions in artificial systems like genetically-modified ORF8 sequences as well as host systems. The issue of independence and invariance is fascinating here. Physiologically-diverse hosts (primate and Rhinolophus bat cells) were measured for replication rate. Using the manipulation indicator, we can see that any manipulation on e.g., bat cells will have no effect on a given sample of primate cells, so there is an indicator of host system independence even in the absence of theory. These measures were compared to replication in non-endemic control hosts (cotton rat, goat, and sheep). Interestingly, Muth et al. (2018) found that $29 \mathrm{nt}$ deletion in ORF8 decreased replication up to 23-fold, invariant over host cell system.

We can use PVM to organize key elements in the measurement process. Multiprocess theory-modification is helpful as an organizational tool:

In context of independent measurement processes, scientists can selectively manipulate certain causal conditions to analyze invariant and varying information for the purpose of modifying assumptions that are the backbone for characterizing variables.

In this case, multi-perspectival, productive, and independent measurements are informative about invariant information in SARS-CoV-2 replication in at least two ways. First, replication can be characterized as a dynamic phenomenon, determined by complex virus-to-host interactions. Three independent studies support SARS-CoV-2 replication-sensitivity-one productive study (Muth et al. 2018), and two epidemiological studies (S.M.E.C. 2004; Su et al. 2020). Second, replication decreases due to specific causal conditions in ORF8 modification. That is, three studies locate the causally-relevant conditions to ORF8. Two studies are based on epidemiological retrospective analysis (S.M.E.C. 2004; Su et al. 2020). The other study makes direct manipulations (Muth et al. 2018) in order to characterize the causal culprit. The Muth et al. (2018) measurements are not just representationally-informative, they are also productively-informative. They provide productive measurement methodology for how to manipulate causal conditions in order to observe the dynamics of replication in different hosts. Moreover, these results are informative because they are independent-i.e. they pass the manipulation indicator test. Combined with the independent conclusion from the Su et al. (2020) study, an implicit theoretical prediction emerges - with prolonged host interaction, ORF8 will be modified, naturally, for up to a 23-fold decrease in replication. Adding another independent measure, S.M.E.C. (2004) observed that towards the end of the SARS outbreak, many cases possessed an ORF8 deletion. A more explicit theoretical picture begins to emerge: host adaptation will produce an attenuated phenotype of SARS-CoV-2 (Su et al. 2020). What is 
methodologically important here is that PVM tracks key elements-manipulation/ intervention, independence, invariance, and theory-development-by organizing unfolding measurement processes. PVM picks out key interactions in the total measurement process - such as, the interaction between virus and host. It also differentiates between productive and representational measurements as well as invariant and varying information. All of this can be used to paint a clear narrative of a given measurement process.

\subsection{COVID-19 measurement prescription}

A blunt question emerges for conceptual developments in measurement theory: So what? In more detail, can a theory of measurement actually help scientific practice? Telling a retrospective story about measurement processes is an interesting analytical exercise; but prospective navigation in measurement practice is currently needed. In this section, we discuss normative points for measurement practice. To adequately account for changing interactions within the context of the pandemic, measurement practices should widen their perspectival scope. Failure to acknowledge the complex measurement interactions and productions, that are relevant to measurement outcomes, can result in impeded measurement and theory-building practices.

In the previous section, we argued that without understanding virus-to-host interactions, we cannot build more robust perspectives on SARS-CoV-2 replication. By accounting for manipulations, interactions, and productions, a comprehensive theory of measurement can explicate the measurement processes for complex, relational phenomena like transmission, replication, morbidity, mortality, etc. Further, a comprehensive theory of measurement can promote the development of new methods that focus on the details of interaction, manipulation, and production.

In Section 3.1, we presented the view that the re-arrangement of conditions and the manipulation of processes, involved in productive measurements, are not necessarily human-centric activities. ${ }^{21}$ That is, causal conditions that are relevant to the measurement process can be altered by scientists; but those conditions can also be altered by things in nature. The re-arrangement of interactions by SARSCoV-2 was presented in the previous section in our discussion about virus-to-host relations. This way, the pandemic can be represented as a context or an interface that reorganizes interactions. As some conditions stabilize both in the lab and in the world to produce, e.g., a repeatable replication rate, it is important not to idealize how those conditions figure in to the total process of measurement. (Recall from Section 2 that the view "from above" often leaves out explicit details of measurement processes. The suggestion is to maintain the "view from within" practice in order to modify it.) Similarly, in the context of the pandemic other conditions destabilize; and understanding precisely how the destabilizing interaction works will be relevant for understanding how measurement outcomes are produced.

\footnotetext{
${ }^{21}$ Recall that van Fraassen's engines of creations and 'productive measurements' in general are determined by measurement conditions that consistently create a given regularity — whether in the lab or in the world.
} 
Currently $^{22}$, many interactions in measurement processes that are causally relevant for SARS-CoV-2/COVID-19 measurement outcomes have been ignored or not properly acknowledged. These "unacknowledged interactions" are not just biological-e.g. the replication rate discussed in Section 4.1 - but they are also related to the built environment, social factors, and health factors. We make suggestions about how to expand the scope of measurement practice to focus on complex interactions between biotic, social, built, and health conditions. When these interactions are left out, inadequate assumptions and theories emerge.

There is a lack of adequate measurement practice to account for disparities in COVID-19 morbidity, mortality, and disproportionate effects based on social systemic factors. Current research is just beginning to unravel the relationship between COVID19 outcomes, built environment factors (e.g., pollution exposure), resource access, various forms of systemic racism, and countless causal connections between biotic, built, social, and health processes-but with severely limited measurement focus on interactions, like how systemic racism influences health outcomes within the context of the pandemic. ${ }^{23}$ Notice here that both the context of the pandemic and systemic factors can reorganize causally relevant conditions - built, biotic, social, health, etc. The concept of 'production' presented in Section 3 is useful. What conditions and interactions are relevant to the production of a given measurement outcome? That is, we are not measuring morbidity and mortality pure and simple. Measurements occur within shifting contexts, where conditions are constantly reorganizing. Does measurement practice track how biotic, built, and social pathways are reorganizing morbidity and mortality outcomes? A theory of measurement can refocus the practice to emphasize complex, dynamically-shifting interactions that are causally relevant to measurement outcomes.

Production is not the only issue. Selective scientific representation in current measurement practice inadequately accounts for the phenomena. Washington (2020) presents the need to adequately represent race in COVID-19 data in order to be able to analyze the connections between environmental racism and pandemic health outcomes. The need is currently unmet, which results in measurement outcomes that do not represent the complexities of the pandemic. Many U.S. cities have not released pandemic morbidity and mortality rates based on race and ethnicity (Brandt et al. 2020). Current studies that link long-term exposure of $\mathrm{PM}_{2.5}, \mathrm{PM}_{10}, \mathrm{NO}_{2}$, and $\mathrm{CO}$ to decreased COVID-19 outcomes (Pansini and Fornacca 2020; Fattorini and Regoli 2020 and $\mathrm{Wu}$ et al. 2020) do not detail race, ethnicity, and systemic factors. Brandt et al. (2020) hypothesize that structural racism produces disparate exposure to air pollution, and they state that complex causal factors - e.g., how specific systemic factors influence pollution exposure - need to be investigated. Current measurement practice has a narrow scope of the interactions that are necessary for COVID-19 health measures.

COVID-19 measurement methodology is missing important, but unacknowledged, interactions between the pandemic, social and systemic processes, built environment processes, and health outcomes. For instance, significant findings based on data from

\footnotetext{
22 Section 4 was developed in March, 2020.

${ }^{23}$ One might argue that such complexity is reserved for theory. But, as will become apparent, an initial failure occurs at the stage of measurement, by ignoring representational information that could serve as the foundation for theory-building.
} 
3000 US counties, predict that with a $1 \mu \mathrm{g} / \mathrm{m}^{3}$ increase in chronic $\mathrm{PM}_{2.5}$ exposure, COVID-19 mortality rate increases by $15 \%$ (Wu et al. 2020). But this is uninformative about the dynamical relations that take place within a given built environment. Brandt et al. (2020) discuss the need to factor in new types of measures about accumulated lifetime exposure to explain why the temporary decrease of $\mathrm{PM}_{2.5}$ will not alleviate health outcomes. But if measurement practice broadens the set of interactions, relevant to COVID-19 measures, it becomes apparent that it is necessary to measure not only accumulated $\mathrm{PM}_{2.5}$ exposure, but also how the built environments mediates pollution, virus particle exposure, as well as lack of health-promoting bacteria.

Often, scientific discipline measurement practices are siloed, which prevents the measurement of important intersections of conditions. In measurement practices pertaining to pandemic airflow, virus particle transmission might be tracked, while microbiota diversity is ignored. It is often not acknowledged that even at ideal function, central HVAC systems can create unsuitable health environments because they eliminate diversity of bacteria and create human disease-centered colonies (Kembel et al. 2012). Furthermore, there is important research that shows asthma as a function of low exposure to beneficial genera-e.g., Lachnospira, Veillonella, Faecalibacterium, and Rothia (Arrieta et al. 2015). Recent work on airflow measurement in the context of the pandemic has fully ignored bacteria measurements. The pandemic context reveals that such intersections need to be measured, so that more robust theories about health resilience in the context of the pandemic can be built.

The obvious criticism might be, but there is no need to broaden the perspectival scope over measurement interactions because scientists are already doing thisindependent measurements are combined; information is synthesized; and larger sets of interactions are theoretically-developed. First, as discussed, measurements are representationally lacking in important parameters, which implies that doing more of that kind of measurement will just produce an amalgam of limited measurements, which will lead to inadequate theory-building activities. Second, there are different kinds of measurement frameworks and methodologies that can be developed by taking an interaction- or relation-based measurement foundation. For instance, interdisciplinary and transdisciplinary measurement practice can be used to measure relations between various forms of systemic racism, built environments, biotic airflow processes, and epidemiology. Research efforts that combine methods and assumptions can explore intersections between sets of physical interactions. Those sets of interactions are often isolated in measurement practices within particular disciplines. The methodological need is to develop new measurement methods and collaborations between scientists, but also, to engage the public to participate in measurement practice.

Public participation in measurement practice can be modeled based on an iterative approach to engaged community measurement practices for the pursuit of environmental justice (Gabrys et al. 2016). Due to a severe deficiency in air quality monitoring infrastructure, Gabrys et al. (2016) proposed a new framework for public data-production and organization. A similar innovative approach can be applied to communitybased COVID-19 data-generation. The necessity and value of qualitative measurementgeneration is a developing topic, especially in the context of public health (Hanlon et al. 2011), where it was previously unrecognized. If community members can autonomously make and report qualitative measurements to scientists and policy-makers, this could potentially create broader measurement perspectives that focus on new types of 
interactions. Additionally, it can change the question - who measures? In Section 2, we abstractly discussed the importance of the "user" in measurement practice to decide the respects and degrees of measurement perspective. The "user" implicitly references the experimenter or theoretician. But the user of measurement practice can also be the public. This conceptual shift promotes social autonomy in measurement practice: the "storying" process is an iterative practice of making and curating measurements (Gabrys et al. 2016); and so, communities can use their storying methods to inform the development of public health measurement perspectives.

\section{Concluding remarks}

We began the discussion with two general questions for a robust account of perspectival measurement: What does the physical interaction of measurement consist of? Moreover, what sort of information about the target of measurement is generated during the measurement interaction? We structured van Fraassen's (2008) view of measurement interaction and information into a set of useful features for understanding the role of perspective in measurement; and then we extended the discussion to be applicable to developing measurement processes. One purpose of this discussion is to show how PVM tracks key elements - manipulation/intervention, independence, invariance, and theory-development-by organizing unfolding measurement processes. But during the development of PVM, the pandemic context put pressure on philosophical theory to be adequate for developing scientific practice. We suggest that PVM can be also be used to frame suggestions for current measurement practices, due to their inadequacies in accounting for relevant interactions between biotic, built, social, and health factors.

\section{References}

Arrieta, M.-C., Stiemsma, L. T., Dimitriu, P. A., Thorson, L., Russell, S., Yurist-Doutsch, S., et al. (2015). Early infancy microbial and metabolic alterations affect risk of childhood asthma. Science Translational Medicine, 7(307), 307ra152. https://doi.org/10.1126/scitranslmed.aab2271.

Berka, K. (1983). Measurement. It's concepts, theories and problems. Dordrecht: Reidel.

Brandt, E. B., Beck, A. F., \& Mersha, T. B. (2020). Air pollution, racial disparities, and COVID-19 mortality. The Journal of Allergy and Clinical Immunology, 146(1), 61-63. https://doi.org/10.1016/j.jaci.2020.04.035.

Campbell, N. R. (1920). Physics: The elements. London: Cambridge University Press.

Chang, H. (2004). Inventing temperature. Oxford: Oxford University Press.

Chen, J., Jiang, Q., Xia, X., Liu, K., Yu, Z., Tao, W., Gong, W., \& Han, J. J. (2020). Individual variation of the SARS-CoV2 receptor ACE2 gene expression and regulation. Preprints, 2020030191.

Culp, S. (1994). Defending robustness: The bacterial Mesosome as a test case. In D. Hull, M. Forbes, \& R. Burian (Eds.), PSA 1994 (pp. 47-57). Philosophy of Science Association: East Lansing.

Doty, R. L. (2010). The great pheromone myth: Mammalian pheromones, Audiomones, Visuomones and Snarks. Baltimore: John Hopkins University Press.

Dorries, K. M., Adkins-Regan, E., \& Halpern, B. P. (1997). Sensitivity and behavioral responses to the pheromone androstenone are not mediated by the vomeronasal organ in domestic pigs. Brain Behavior Evolution, 49(1), 53-62.

Ellis, B. (1966). Basic concepts of measurement. London: Cambridge University Press.

Fattorini, D., \& Regoli, F. (2020). Role of the chronic air pollution levels in the Covid-19 outbreak risk in Italy. Environmental Pollution, 264, 114732. https://doi.org/10.1016/j.envpol.2020.114732.

Franklin, A. (1997). Calibration. Perspectives on Science, 5, 31-80. 
Gabrys, J., Pritchard, H., \& Barratt, B. (2016). Just good enough data: Figuring data citizenships through air pollution sensing and data stories. Big Data \& Society, 3(2), 2053951716679677. https://doi.org/10.1177/ 2053951716679677.

Giere, R. N. (2006). Scientific Perspectivism. Chicago: The University of Chicago Press.

Hanlon, P., Carlisle, S., Hannah, M., Reilly, D., \& Lyon, A. (2011). Making the case for a 'fifth wave' in public health. Public Health, 125(1), 30-36 https://doi.org/10.1016/j.puhe.2010.09.004.

Heidelberger, M. (2003). Theory-ladenness and scientific instruments. In H. Radder (Ed.), The philosophy of scientific experimentation (pp. 138-151). Pittsburgh: University of Pittsburgh Press.

Helmholtz, H. V. (1887). Zählen und messen erkenntnis-theoretisch betrachet, in H. V. Helmholtz, Schriften zur Erkenntnistheorie, pages 70-108. English translation, numbering and measuring from an epistemological viewpoint, in H. V. Helmoltz, Epistemological Writings, pp. 72-114. Dordrecht: Reidel

Horwich, P. (1982). Probability and evidence. Cambridge: Cambridge University Press.

JCGM (Joint Committee for Guides in Metrology). (2008). Guide to the Expression of Uncertainty in Measurement (GUM). Published by the Joint Committee for Guides in Metrology, 3rd edition. Sèvres: http://www.bipm.org/en/publications/guides/gum.html.

JCGM (Joint Committee for Guides in Metrology). (2012). International Vocabulary of Metrology. 3th edition.

Keeley, B. (2002). Making sense of the senses: Individuating modalities in humans and other animals. The Journal of Philosophy, 99(1), 5-28.

Kembel, S. W., Jones, E., Kline, J., Northcutt, D., Stenson, J., Womack, A. M., Bohannan, B. J., Brown, G. Z., \& Green, J. L. (2012). Architectural design influences the diversity and structure of the built environment microbiome. The ISME Journal, 6(8), 1469-1479. https://doi.org/10.1038/ismej.2011.211.

Krantz, D. (1964). Conjoint measurement: The lute-tukey axiomatization and some extensions. Journal of Mathematical Psychology, 1, 248-277.

Kyburg, H. (1984). Theory and Measurement. Cambridge: Cambridge University Press.

Lloyd, E. A. (2015). Model robustness as a confirmatory virtue: The case of climate science. Studies in History and Philosophy of Science Part A, 49, 58-68.

Luce, R. D., \& Narens, L. (1994). Fifteen problems concerning the representational theory of measurement. In P. Humphreys (Ed.), Patrick Suppes: Scientific philosopher, Philosophy of physics, theory structure, measurement theory, philosophy of language, and logic (Vol. 2, pp. 219-245). Dordrecht: Kluwer Academic Publishers.

Massimi, M., \& McCoy, D. C. (2018). Understanding perspectivism: Scientific challenges and methodological prospects. London: Routledge.

Muth, D., Corman, V. M., Roth, H., Binger, T., Dijkman, R., Gottula, L. T., Gloza-Rausch, F., Balboni, A., Battilani, M., Rihtarič, D., Toplak, I., Ameneiros, R. S., Pfeifer, A., Thiel, V., Drexler, J. F., Müller, M. A., \& Drosten, C. (2018). Attenuation of replication by a 29 nucleotide deletion in SARS-coronavirus acquired during the early stages of human-to-human transmission. Scientific Reports, 8, 15177. https:// doi.org/10.1038/s41598-018-33487-8.

Nagel, E. (1930). On the logic of measurement. Stanford: Stanford University Press.

Narens, L. (1985). Abstract measurement theory. Cambridge, MA: MIT Press.

Pansini, R., \& Fornacca, D. (2020). Initial evidence of higher morbidity and mortality due to SARS-CoV-2 in regions with lower air quality. MedRxiv, 2020(04), 04.20053595. https://doi.org/10.1101/2020.04.04. 20053595.

Pfanzagl. (1968). Theory of measurement. New York: Wiley.

Prasolova, L. A., Trut, L. N., Os'kina, I. N., Gulevich, R. G., Pliusnina, I. Z., Vsevolodov, E. B., \& Latypov, I. F. (2006). The effect of methyl-containing supplements during pregnancy on the phenotypic modification of offspring hair color in rats. Genetika, 42(1), 78-83.

Rasmussen, N. (2001). Evolving scientific epistemologies and the artifacts of empirical philosophy of science: A reply concerning mesosomes. Biology and Philosophy, 16, 629-654.

Reichenbach, H. (1965). The theory of relativity and a priori knowledge. Tr. Maria Reichenbach. Berkeley: University of California Press.

Roberts, F. (1979). Measurement theory. Reading, MA: Addison-Wesley.

Schupbach, J. N. (2016). Robustness analysis as explanatory reasoning. British Journal for the Philosophy of Science, 69(1), 275-300.

Sober, E. (1989). Independent evidence about a common cause. Philosophy of Science, 56, 275-287.

Sokolowska, M., Frei, R., Lunjani, N., Akdis, C. A., \& O’Mahony, L. (2018). Microbiome and asthma. Asthma Research and Practice, 4, 1-1. PubMed. https://doi.org/10.1186/s40733-017-0037-y.

Staley, K. (2004). Robust evidence and secure evidence claims. Philosophy of Science, 71, 467-488.

Stegenga, J. (2009). Robustness, discordance, and relevance. Philosophy of Science, 76(5), 650-661. 
Stegenga, J. (2012). Rerum Concordia Discors: Robustness and discordant multimodal evidence. In Characterizing the Robustness of Science, Boston Studies in the Philosophy of Science, Springer, pp. 207-226.

Stevens, S. S. (1946). On the theory of scales of measurement. Science, 103(2684), 667-680.

Su, Yvonne C. F., Danielle E. Anderson, Barnaby E. Young, Martin Linster, Feng Zhu, Jayanthi Jayakumar, Yan Zhuang, et al. (2020) "Discovery and Genomic Characterization of a 382-Nucleotide Deletion in ORF7b and ORF8 during the Early Evolution of SARS-CoV-2." Edited by Stacey Schultz-Cherry. MBio 11(4): e01610-e01620. https://doi.org/10.1128/mBio.01610-20.

Suppes, P., \& Zinnes, J. L. (1963). Basic measurement theory. In R. D. Luce, R. R. Bush, \& E. H. Galanter (Eds.), Handbook of mathematical psychology (Vol. 1, pp. 1-76). New York: Wiley.

S.M.E.C. (2004). Molecular evolution of the SARS coronavirus during the course of 248 the SARS epidemic in China. Science, 303(5664), 1666-1669.

Tal, E. (2013). Old and new problems of measurement. Philosophical Compass, 8(12), 1159-1173.

Teller, P. (2018). Referential and perspectival realism. Spontaneous Generations: A Journal for the History and Philosophy of Science, 9(1), 151-164.

Trout, J. D. (1998). Measuring the intentional world. Oxford: Oxford University Press.

van Fraassen, B. C. (2008). Scientific representation: Paradoxes of perspective. Oxford: Oxford University Press.

Waldminghaus, T., Heidrich, N., Brantl, S., \& Narberhaus, F. (2007). Lehrstuhl für biologie der mikroorganismen. Molecular Microbiology, 65(2), 413-424.

Washington, H. (2020). How environmental racism is fuelling the coronavirus pandemic. Nature, 581(7808), 241. https://doi.org/10.1038/d41586-020-01453-y.

Wimsatt, W. (2007). Re-engineering philosophy for limited beings: Piecewise approximations to reality. Cambridge: Harvard University Press.

Woodward, J. (2003). Making things happen: A theory of causal explanation: Oxford University press, USA. Woodward, J. (2006). Some varieties of robustness. Journal of Economic Methodology, 13(2), 219-240.

Wu, C., Chen, X., Cai, Y., Xia, J., Zhou, X., Xu, S., Huang, H., Zhang, L., Zhou, X., Du, C., Zhang, Y., Song, J., Wang, S., Chao, Y., Yang, Z., Xu, J., Zhou, X., Chen, D., Xiong, W., et al. (2020). Risk factors associated with acute respiratory distress syndrome and death in patients with coronavirus disease 2019 pneumonia in Wuhan, China. JAMA Internal Medicine, 180(7), 1-11. https://doi.org/10.1001/ jamainternmed.2020.0994.

Publisher's note Springer Nature remains neutral with regard to jurisdictional claims in published maps and institutional affiliations. 